OSU-TA-12/94

\title{
GENERIC EVOLUTION OF DEUTERIUM AND HELIUM-3
}

\author{
GARY STEIGMAN \\ Departments of Physics and Astronomy \\ The Ohio State University \\ 174 West 18th Avenue, Columbus, OH 43210, USA \\ and \\ MONICA TOSI \\ Osservatorio Astronomico di Bologna, \\ Via Zamboni 33, I-40126 Bologna, ITALY
}

\begin{abstract}
Summary
The primordial abundances of deuterium and of helium-3, produced during big bang nucleosynthesis, depend sensitively on the baryon density. Thus, the observed abundances of D and ${ }^{3} \mathrm{He}$ may provide useful "baryometers" provided the evolution from primordial to present (or, presolar nebula) abundances is understood. Inevitably, the derivation of primordial from observed abundances requires the intervention of a model for galactic evolution and, so, the inferred primordial abundances are, necessarily, model dependent. Here, an analytic framework for the evolution of $\mathrm{D}$ and ${ }^{3} \mathrm{He}$ is presented which is "generic" in the sense that it should describe the results of any specific galactic evolution model. The "effective ${ }^{3}$ He survival fraction", $\Gamma_{3}$, is the one free parameter which is model specific. Solar system and interstellar data are used to infer upper and lower bounds to the primordial deuterium mass fraction $\left(X_{2 P}\right)$ as a function of $\Gamma_{3}$ and, these bounds are used to constrain the present baryon-to-photon ratio $(\eta)$ and baryon density $\left(\Omega_{B}\right)$. For $\Gamma_{3} \geq 1 / 4$ it is found that (from $\mathrm{D}$ and ${ }^{3} \mathrm{He}$ alone): $3.1 \leq \eta_{10} \leq 9.0$; $0.045 \leq \Omega_{B} h_{50}^{2} \leq 0.133$ (where $H_{0}=50 h_{50} \mathrm{~km} \mathrm{~s}^{-1} \mathrm{Mpc}^{-1}$ ).
\end{abstract}




\section{Introduction}

The light nuclides $\mathrm{D},{ }^{3} \mathrm{He},{ }^{4} \mathrm{He}$ and ${ }^{7} \mathrm{Li}$ are produced in astrophysically interesting abundances during big bang nucleosynthesis (BBN) and, hence, their primordial abundances provide quantitative tests of the standard hot big bang model. However, primordial abundances are not observed but, rather, derived from observational data and - to a greater or lesser extent - models for galactic chemical evolution. Since ${ }^{4} \mathrm{He}$ and ${ }^{7} \mathrm{Li}$ are observed in metal-poor objects (extragalactic HII regions for the former, PopII stars for the latter), the evolutionary corrections are minimized. Unfortunately, this is not the case for D and ${ }^{3} \mathrm{He}$ where observations are, for the most part, limited to the solar system and the present interstellar medium (ISM). The inevitable intervention of galactic evolution models results in model dependent uncertainties for the primordial abundances of D and ${ }^{3} \mathrm{He}$ derived from even the most accurate observational data. Indeed, for this reason the most fruitful approach has been to use the observational data to bound the primordial abundances. For example, since deuterium is only destroyed during the course of galactic evolution, the primordial abundance must exceed the observed abundance $\left(X_{2 P} \geq X_{2 \text { OBS }}\right.$ where $X_{2 \mathrm{OBS}}=X_{2 \odot}$ or $\left.X_{2 \mathrm{ISM}}\right)$. It has, however, proved harder to use the observational data to bound $X_{2 P}$ from above (Yang et al. 1984, YTSSO). YTSSO and Dearborn, Schramm \& Steigman (1986, DSS) first noted that since D burns to ${ }^{3} \mathrm{He}$ and some ${ }^{3} \mathrm{He}$ survives stellar evolution, the observed abundances of $\mathrm{D}$ and ${ }^{3} \mathrm{He}$ may be used to bound, from above, the primordial abundance of $\mathrm{D}+{ }^{3} \mathrm{He}$. The upper bound to the primordial abundance of $\mathrm{D}$ $+{ }^{3} \mathrm{He}$ derived by YTSSO, and utilized in many subsequent analyses, depends on the ${ }^{3} \mathrm{He}$ survival fraction $g_{3}$ which DSS computed from models for the evolution of stars of different masses. However, unlike the model-independent lower bound

$\left(X_{2 P} \geq X_{2 \mathrm{OBS}}\right)$, this upper bound to $\mathrm{D}+{ }^{3} \mathrm{He}$ is still model dependent in the sense that the "effective" value of $g_{3}$, hereafter indicated by $\Gamma_{3}$ to distinguish it from the DSS $g_{3}$, depends not only on stellar structure/evolution but, also, on the past star formation history, the IMF (and its evolution), infall and outflow, etc. In short, since the "true" value of $\Gamma_{3}$ is model dependent, so too is the upper bound to the primordial abundance of deuterium derived from observations of $\mathrm{D}$ and ${ }^{3}$ He. Indeed, using very much the same stellar data, Steigman \& Tosi (1992, ST92) find deuterium destruction factors $\leq 2-3$ while Vangioni-Flam \& Audouze (1988) and Vangioni-Flam, Olive \& Prantzos (1994) find destruction factors as large as $\sim 5-10$ are allowed and, the more extreme models of Vangioni-Flam \& Cassé (1994) permit even more D destruction. We note that these latter models have been designed to account for the D data and they may not necessarily be consistent with other observational constraints on the abundances, abundance ratios, and gradients of the other elements. Indeed, Edmunds (1994) argues on the 
basis of "Simple Model" predictions, that they may not be consistent with the age-metallicity relation and the G-dwarf metallicity distribution.

Despite the above-cited model dependent uncertainties, it is important to pursue the primordial abundance of deuterium. The main reason is that, in the context of $\mathrm{BBN}, X_{2 P}$ (the primordial deuterium mass fraction) is a sensitive "baryometer". At higher nucleon (baryon) densities D burns faster (to ${ }^{3} \mathrm{H}$, ${ }^{3} \mathrm{He},{ }^{4} \mathrm{He}$ ) and less deuterium emerges from BBN. $X_{2 P}$ is a steeply decreasing function of the nucleon-to-photon ratio $\eta\left[\eta_{10}=10^{10}(\mathrm{~N} / \gamma)\right]$; for $2 \leq \eta_{10} \leq 6$, $X_{2 P} \sim \eta_{10}^{-1.7}$. Thus, an upper (lower) bound to $X_{2 P}$ will lead to a lower (upper) bound to $\eta$ and, hence, to bounds on the current universal density of baryons $\left(\Omega_{B}=\rho_{B} / \rho_{\text {crit }}=0.015 \eta_{10} h_{50}^{-2}\right.$ where $\left.H_{0}=50 h_{50} \mathrm{~km} \mathrm{~s}^{-1} \mathrm{Mpc}^{-1}\right)$. Furthermore, a lower bound to $\eta$ (derived from an upper bound to $X_{2 P}$ ), when combined with an upper bound to the primordial ${ }^{4} \mathrm{He}$ mass fraction, can be used to bound the "effective" number of equivalent light neutrino degrees of freedom (Steigman, Schramm \& Gunn 1977) and, hence, to constrain "new" physics (beyond the standard model of the strong and electroweak interactions). Thus, rather than ignore primordial deuterium as a potential baryometer, it is surely more worthwhile to try to understand the nature and extent of the model dependent uncertainties in its inferred primordial abundance. To this end, it is the goal here to derive simple analytical relations between the primordial and observed abundances of $\mathrm{D}$ and ${ }^{3} \mathrm{He}$ which are general in the sense that they do not rely on the special details of any specific chemical evolution model. The equations to be derived in the next section are "generic" but, they depend on one model dependent parameter, $\Gamma_{3}$, the "effective survival fraction of ${ }^{3} \mathrm{He}$ ". Different galactic evolution models will yield different values of $\Gamma_{3}$ (as a function of time and of location in the Galaxy). Highlighting the physical reasons for the various values of $\Gamma_{3}$ may aid in identifying a "reasonable" range for $\Gamma_{3}$ and, hence, lead to nearly model independent bounds on $X_{2 P}, \eta_{10}$ and $\Omega_{B}$.

\section{Evolution of Deuterium and Helium-3}

At any time $(t)$ consider a "representative" volume $(V)$ of the ISM which, initially, contained an amount of gas $M_{i}$. In general, the gas mass in $V$ will change with time due to infall, outflow and the sequestering (even if temporary) of gas in stars. At any time during the evolution of the Galaxy there will be a fraction, $f(t)$, of the gas in the ISM (in $V$ ) which has never been through stars. The remaining fraction, $1-f(t)$, has been cycled through stars - one or more times - and returned to the gas. Thus, at time $t$ in volume $V, f(t) M(t)$ has never been through stars and $(1-f(t)) M(t)$ has been through one or more generations of stars. Further, of the amount of gas in $V$ at $t, M(t)$, there were initially (prior to any evolution) 
$N_{2 i}$ deuterium nuclei where,

$$
N_{2 i}=\left(X_{2 i} / 2\right) M_{i} / M_{N} .
$$

In (1), $X_{2 i}$ is the initial (primordial) D mass fraction and $M_{N}$ is the nucleon mass. Since any $\mathrm{D}$ cycled through stars is destroyed, at time $t$ in volume $V$ only $N_{2}(t) D$-nuclei remain.

$$
N_{2}(t)=f(t)\left(X_{2 i} / 2\right)(M(t)) / M_{N} .
$$

Since $X_{2}(t)=2 N_{2}(t) M_{N} / M(t)$, the D-survival factor $\left(f_{2}\right)$ and the virgin gas fraction $(f)$ are identical.

$$
f_{2}(t) \equiv X_{2}(t) / X_{2 i}=f(t) .
$$

Since deuterium is only destroyed, $f_{2}(t) \leq 1$.

Next, consider the evolution of ${ }^{3} \mathrm{He} .{ }^{3} \mathrm{He}$ is destroyed in the hot interiors of all stars but preserved in the cooler outer layers (Iben 1967; Rood 1972). For cooler low mass stars $\left(\leq 2.5 \mathrm{M}_{\odot}\right)$ hydrogen burning results in the net production of ${ }^{3} \mathrm{He}$ (Iben 1967; Rood 1972; Rood, Steigman \& Tinsley 1976; DSS). Although net stellar production of ${ }^{3} \mathrm{He}$ is potentially large, especially in the epoch between the birth of the solar system and the present, when the most effective ${ }^{3} \mathrm{He}$ producers start to contribute to the ISM enrichment, it is also very uncertain (Rood, Steigman \& Tinsley 1976; DSS; Galli et al. 1994, Hogan 1994, Tosi et al. 1994). Thus, in the spirit of deriving "conservative" upper bounds to the primordial abundances of D and ${ }^{3} \mathrm{He}$, newly synthesized ${ }^{3} \mathrm{He}$ (as distinct from prestellar D burned to ${ }^{3} \mathrm{He}$ ) is ignored in the following. This will lead to a lower bound to $X_{3}(t)$, the ${ }^{3} \mathrm{He}$ mass fraction at any time $t$. The quantitative effect of this neglect is reduced if presolar nebular abundances are used rather than current interstellar values. Now, since a fraction $f(t)$ of the ${ }^{3} \mathrm{He}$ nuclei which were originally in $M(t)$ have never been in stars and a fraction $1-f(t)$ have been cycled through stars, for $X_{3}(t)=3 N_{3}(t) M_{N} / M(t)$ it follows that,

$$
X_{3}(t) \geq X_{3 i} f(t)+(1-f(t)) \Gamma_{3}(t)\left[X_{3 i}+3 X_{2 i} / 2\right] .
$$

In (4), $\Gamma_{3}(t)$ is the "effective survival fraction" of ${ }^{3} \mathrm{He} . \Gamma_{3}$ is model and time dependent since it depends not only on the stellar models but, on the IMF and on the history of star formation (e.g., which stars have returned their material to the ISM by time $t$ ). In addition, although in material going through stars for the first time the prestellar ${ }^{3} \mathrm{He}$ abundance is the sum of the $\mathrm{D}$ and ${ }^{3} \mathrm{He}$ primordial abundances ( $\mathrm{D}$ is immediately burned to ${ }^{3} \mathrm{He}$ ), any material going through stars 
for a second (or more) time will have had its initial deuterium already destroyed; this, too, modifies $\Gamma_{3}$ from the simple DSS values $g_{3}$ and, thus, $\Gamma_{3}$ depends on how much of the gas that has been through stars at least once has been through subsequent generations.

The ${ }^{3}$ He evolution factor $f_{3}(t)$ is,

$$
f_{3}(t) \equiv X_{3}(t) / X_{3 i} \geq f(t)+(1-f(t)) \Gamma_{3}(t)\left(y_{23 i} / y_{3 i}\right) .
$$

In (5), $y_{23 i} / y_{3 i}$ is the initial ratio (by number) of $D+{ }^{3} \mathrm{He}$ to ${ }^{3} \mathrm{He}$. By combining equations (3) and (5) we may eliminate $f(t)$ and directly relate $X_{2 i}$ and $X_{3 i}$ to $X_{2}(t)$ and $X_{3}(t)$. However, before doing so, it is instructive to explore those conditions under which $X_{3}$ will increase from its initial value $\left(f_{3}>1\right)$. Eq. (5) may be rewritten as,

$$
f_{3}(t) \geq 1+(1-f(t))\left[\Gamma_{3}(t)\left(y_{23 i} / y_{3 i}\right)-1\right] .
$$

Since $1-f(t) \geq 0$, the ${ }^{3}$ He mass fraction will increase if $\Gamma_{3}(t)>y_{3 i} / y_{23 i}$. As an illustration, if $\Gamma_{3} \geq 1 / 4$ (DSS), $\left(y_{23} / y_{3}\right)_{P} \geq 4$ is required; for standard BBN (Walker et al. 1991, WSSOK) this is satisfied for $\eta_{10} \leq 4.5$. Thus, if $\Gamma_{3 \odot} \geq 1 / 4$ and $\eta_{10} \leq 4.5$, it is predicted that $X_{3 \odot} \geq X_{3 P}\left(\geq 2.9 \times 10^{-5}\right)$; this is (as will be seen) consistent with solar system data (Geiss 1993). Alternatively the ST92 models $^{1}$ suggest that $\Gamma_{3 \odot} \geq 1 / 2$ which implies $X_{3 \odot} \geq X_{3 P}$ for all $\eta_{10} \leq 10$.

The model dependent factor $f_{2}(t)=f(t)$ may be eliminated from (5) by the use of (3), relating the abundances (by number with respect to hydrogen) of D and ${ }^{3} \mathrm{He}$ initially to those at any later time $t$.

$$
y_{2 i} \leq\left(\frac{X}{X_{i}}\right)\left\{\left[y_{23}+\left(\frac{1}{\Gamma_{3}}-1\right) y_{3}\right]-\frac{1}{\Gamma_{3}}\left(\frac{y_{3 i}}{y_{23 i}}\right) y_{23}\right\} .
$$

In (7), $X=X(t)$ is the hydrogen mass fraction and $X_{i}$ is its initial value. The inequality is due to the neglect of stellar synthesis of ${ }^{3} \mathrm{He}$ and is generic to all models of galactic evolution. The only model dependence in $(7)$ is in $\Gamma_{3}(t)$. It will be noticed that eq. (7) is a "mixed" expression in the sense that the last term on the right hand side depends on both initial and final abundances. One approach (YTSSO) is to neglect this term, strengthening the inequality; this is equivalent to assuming zero initial ${ }^{3} \mathrm{He}$. For $y_{3 i}>0$,

$$
y_{2 i}<y_{2 i}^{0} \leq\left(\frac{X}{X_{i}}\right)\left[y_{23}+\left(\frac{1}{\Gamma_{3}}-1\right) y_{3}\right] \text {. }
$$

${ }^{1}$ Due to unfortunate confusion in the DSS definition of $g_{3}$ (which is by mass and not by number), the survival fractions $f_{3}$ published by ST92 were underestimated on average by a factor 1.2 . This implies $\Gamma_{3 \odot} \approx \Gamma_{3 I S M} \approx 0.60$ 
Except for the prefactor $\left(X / X_{i}\right)$, eq. (8) (for $\left.y_{2 i}^{0}\right)$ is precisely the expression derived in YTSSO and used in many subsequent analyses. However, this prefactor is required to account properly for the changing hydrogen mass fraction. Since $X(t)<X_{i}$ the "usual" bound to $y_{2 i}$ is somewhat overestimated; for example, for $X_{P}=0.76 \pm 0.02$ and $X(t)=0.70 \pm 0.02, X(t) / X_{P}=0.92 \pm 0.04$.

The symbiotic relationship between $\mathrm{D}$ and ${ }^{3} \mathrm{He}$ is clear in equations (7) and (8) where, for fixed (observed) abundances $y_{2}$ and $y_{3}$, the initial abundances of $\mathrm{D}$ and ${ }^{3} \mathrm{He}$ are anticorrelated (e.g., higher $y_{3 i}$ forces lower $y_{2 i}$ ). Although eq. (8) provides a valuable BBN-independent bound to pre-galactic deuterium (Steigman 1994), the last term on the right hand side of (7) should not, in general, be neglected. This poses no problem in practice since, for a specific BBN model the left and right hand sides of (7) may be compared to find if the inequality is, or is not, satisfied. In this manner the observational data can be used, along with evolution model-dependent choices of $\Gamma_{3}$, to constrain the models/parameters of BBN.

In subsequent comparisons, the solar system data of Geiss (1993) will be adopted. The observed quantities, derived from solar wind, lunar and meteoritic data are the ratios (by number) to ${ }^{4} \mathrm{He}$ of presolar $\mathrm{D}+{ }^{3} \mathrm{He}$ and ${ }^{3} \mathrm{He}\left(y_{23} / y_{4}\right.$ and

$\left.y_{3} / y_{4}\right)$. With such data it is convenient to rewrite (7) and (8) in the following forms,

$$
\begin{gathered}
X_{2 i} \leq \frac{Y_{\odot}}{2}\left\{\left[1-\frac{1}{\Gamma_{3 \odot}}\left(\frac{y_{3 i}}{y_{23 i}}\right)\right]\left(\frac{y_{23}}{y_{4}}\right)_{\odot}+\left(\frac{1}{\Gamma_{3 \odot}}-1\right)\left(\frac{y_{3}}{y_{4}}\right)_{\odot}\right\}, \\
X_{2 i}^{0} \leq \frac{Y_{\odot}}{2}\left[\left(\frac{y_{23}}{y_{4}}\right)_{\odot}+\left(\frac{1}{\Gamma_{3 \odot}}-1\right)\left(\frac{y_{3}}{y_{4}}\right)_{\odot}\right] .
\end{gathered}
$$

In (9) and (10), $Y_{\odot}$ is the solar ${ }^{4} \mathrm{He}$ mass fraction.

\section{Solar System Abundances}

In a very valuable recent review, Geiss (1993) has reanalysed the solar system data crucial to determining the presolar nebular abundances of $\mathrm{D}$ and ${ }^{3} \mathrm{He}$. Although Geiss' central values are virtually identical to those used in the past (e.g., Boesgaard \& Steigman 1985), his error bars are more generous. Here, with a little care towards error propagation, the Geiss (1994) analysis is adopted.

$$
\begin{aligned}
& \left(y_{23} / y_{4}\right)_{\odot}=4.09 \pm 0.83 \times 10^{-4} \\
& \left(y_{3} / y_{4}\right)_{\odot}=1.52 \pm 0.30 \times 10^{-4} .
\end{aligned}
$$


The adopted value of the solar ${ }^{4} \mathrm{He}$ abundance, with generous allowance for uncertainty, is

$$
Y_{\odot}=0.28 \pm 0.02 \quad\left(y_{4 \odot}=0.10 \pm 0.01\right) .
$$

For $Z_{\odot} \approx 0.02, X_{\odot}=0.70 \pm 0.02$ and the above values may be used to derive:

$$
\begin{aligned}
\left(Y_{\odot} / 2\right)\left(y_{23} / y_{4}\right)_{\odot} & =5.73 \pm 1.23 \times 10^{-5} \\
\left(Y_{\odot} / 2\right)\left(y_{3} / y_{4}\right)_{\odot} & =2.13 \pm 0.45 \times 10^{-5} \\
X_{2 \odot} & =3.60 \pm 1.26 \times 10^{-5} \\
X_{3 \odot} & =3.19 \pm 0.67 \times 10^{-5} \\
y_{23 \odot} & =4.09 \pm 0.92 \times 10^{-5} \\
y_{3 \odot} & =1.52 \pm 0.34 \times 10^{-5} \\
y_{2 \odot} & =2.57 \pm 0.92 \times 10^{-5}
\end{aligned}
$$

\section{Bounds To Primordial Deuterium}

Armed with solar system abundances, equations (3) and (9) (or (10)) may be enlisted to provide upper and lower bounds to the pregalactic (primordial) abundance of deuterium. If equation (9) is used, $y_{3 i} / y_{23 i}$ will be taken from standard BBN (e.g., WSSOK). As already emphasized, the only galactic evolution model dependence is contained in $\Gamma_{3 \odot}$, the effective ${ }^{3} \mathrm{He}$ survival fraction in the solar

neighbourhood at the time of the formation of the solar system. For quantitative comparisons $\Gamma_{3 \odot}=1 / 4$ and $\Gamma_{3 \odot}=1 / 2$ will be adopted; in general, the larger $\Gamma_{3 \odot}$, the smaller the upper bound to $X_{2 P}$.

Since, at the time of formation of the solar system, at least some of the material then present in the local ISM had been cycled through stars, $f_{\odot}<1$. Thus, $X_{2 P}>X_{2 \odot}$; this differs from the often quoted constraint $y_{2 P}>y_{2 \odot}$ by the factor $X_{\odot} / X_{P} \approx 0.9$. At the 2-sigma ( $~ 95 \%$ confidence) level, it follows from (13c) that,

$$
X_{2 P}>X_{2 \odot} \geq 1.08 \times 10^{-5} .
$$

Because of the relatively large uncertainty in $X_{2 \odot}$, this only provides a very weak constraint on $X_{2 P}$. For a more restrictive lower bound to primordial deuterium, the ISM D/H ratio (Linsky et al. 1993) is more useful. The interstellar observations from Copernicus and IUE (see, e.g., Boesgaard \& Steigman 1985) and HST (Linsky et al. 1993) extend over two orders of magnitude in $\mathrm{H}$ column density and are consistent with

$$
y_{2 \mathrm{ISM}}=1.6 \pm 0.2 \times 10^{-5} \text {. }
$$


If $X_{\mathrm{ISM}} \approx X_{\odot} \approx 0.70 \pm 0.02$, then at 2-sigma,

$$
X_{2 P}>X_{2 \mathrm{ISM}}>1.7 \times 10^{-5}
$$

For standard BBN (WSSOK) this provides an upper bound to the nucleon density,

$$
\eta_{10}<9.0
$$

where $\eta$ is the ratio of nucleons to photons at present and $\eta_{10} \equiv 10^{10} \eta$.

As an aside, notice that if the solar system (13g) and interstellar (15) D/H ratios are compared,

$$
X_{2 \odot} / X_{2 \mathrm{ISM}} \approx y_{2 \odot} / y_{2 \mathrm{ISM}}=1.6 \pm 0.6
$$

This provides a weak ( 1-sigma) hint that, in the solar neighbourhood, some D has been destroyed in the last 4.6 Gyr.

In considering the upper bound to the primordial deuterium mass fraction from equations (9) or (10), it is useful to compare the BBN predicted value $X_{2 \mathrm{BBN}}$ (as a function of $\eta$ ) with the analytic bounds $X_{2 \mathrm{MAX}}$ (eq. 9) and $X_{2 \mathrm{MAX}}^{0}$ (eq. 10). For a " $2 \sigma$ " upper bound, equations (9) and (10) are evaluated with the $2 \sigma$ upper bounds to the solar system abundances (see $13 \mathrm{a} \& \mathrm{~b}:\left(Y_{\odot} / 2\right)\left(y_{23} / y_{4}\right) \odot \leq$ $\left.8.2 \times 10^{-5},\left(Y_{\odot} / 2\right)\left(y_{3} / y_{4}\right)_{\odot} \leq 3.0 \times 10^{-5}\right)$. The results for $X_{2 \mathrm{MAX}}$ and $X_{2 \mathrm{MAX}}^{0}$ are shown in Figure 1 for $\Gamma_{3}=1 / 4 \& 1 / 2$ respectively. Also shown in Figure 1 is $X_{2 \mathrm{BBN}}$; the requirement that $X_{2 \mathrm{BBN}}<X_{2 \mathrm{MAX}}$ (or $X_{2 \mathrm{MAX}}^{0}$ ) provides a lower bound to $\eta$.

As anticipated, the neglect of any primordial ${ }^{3} \mathrm{He}\left(X_{2 \mathrm{MAx}}^{0}\right)$ provides a weaker constraint than if the $\mathrm{BBN}$ predicted relative ${ }^{3} \mathrm{He} / \mathrm{D}$ abundance is allowed for $\left(X_{2 \mathrm{MAX}}\right)$; for all $y_{3 P}>0, X_{2 \mathrm{MAX}}^{0}>X_{2 \mathrm{MAX}}$. As $\eta$ increases, the BBN yields of $\mathrm{D}$ and ${ }^{3} \mathrm{He}$ decrease but, $\left({ }^{3} \mathrm{He} / \mathrm{D}\right)_{\mathrm{BBN}}$ increases. It is the increasing importance of ${ }^{3} \mathrm{He}$ at high $\eta$ which is responsible for the decrease in $X_{2 \mathrm{MAX}}$.

As more ${ }^{3}$ He survives stellar processing ( $\Gamma_{3}$ increases), consistency with the solar system abundances restricts primordial D to lower values $\left(X_{2 \mathrm{MAX}}\right.$ and $X_{2 \mathrm{MAX}}^{0}$ decrease). Thus, for fixed observed abundances, the lower $\Gamma_{3 \odot}$, the weaker the

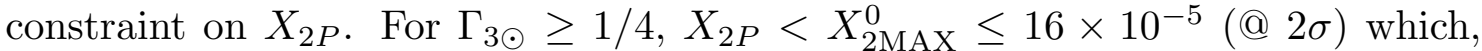
for $\mathrm{BBN}$, corresponds to $\eta_{10}^{0} \geq 2.5$. However, for $\eta_{10} \geq 2.5, y_{3 P} / y_{23 P} \geq 0.14$ and the neglect of primordial ${ }^{3} \mathrm{He}$ is non-negligible. From Figure 1 it may be seen that for $\Gamma_{3 \odot} \geq 1 / 4, X_{2 \mathrm{BBN}}<X_{2 \mathrm{MAX}}(@ 2 \sigma)$ for $X_{2 P} \leq 11 \times 10^{-5}$; this corresponds to $\eta_{10}^{\mathrm{MIN}}>3.1$. For the same solar system data, if $\Gamma_{3 \odot}$ increases to $\gtrsim 1 / 2, \eta_{10}^{\mathrm{MIN}}$ increases to $\gtrsim 4.0$. 
In Figure $2, X_{2 \mathrm{BBN}}$ is compared to the $2 \sigma$ upper bound to $X_{2 \mathrm{MAx}}$ for $\Gamma_{3 \odot}=$ $1 / 4$ and, to the $2 \sigma$ lower bound to $X_{2 \mathrm{MIN}}$ from the interstellar $\mathrm{D} / \mathrm{H}$ ratio (eq. 16). From $\mathrm{D}$ and ${ }^{3} \mathrm{He}$ alone, $\eta$ is then restricted to the range $3.1 \leq \eta_{10} \leq 9.0$. Observational data on ${ }^{4} \mathrm{He}$ and ${ }^{7} \mathrm{Li}$ (e.g. WSSOK; Olive \& Steigman 1994) further constrain the upper bound to $\eta\left(\eta_{10} \leq 3.9\right)$.

Earlier, it had been noted that, provided $\Gamma_{3}$ is not too small nor $y_{3 P} / y_{23 P}$ too large, it is likely that the ${ }^{3} \mathrm{He}$ mass fraction will increase from its primordial value (even neglecting net stellar production of ${ }^{3} \mathrm{He}$ ). Thus, for $\Gamma_{3 \odot} \geq 1 / 4$ and $\eta_{10} \leq 4.5$, it was expected that $X_{3 \odot} \geq X_{3 \mathrm{BBN}}$. This upper bound to primordial ${ }^{3} \mathrm{He}$ will also lead to a lower bound to $\eta$ which, although weaker than that above, is still of some interest. From (13d), @2 $\sigma, X_{3 P} \leq X_{3 \odot} \leq 4.5 \times 10^{-3}$, which corresponds to $\eta_{10} \geq$ 2.0. Since this bound is independent of the previous deuterium constraint it may be used to bound primordial/pregalactic D: for $\eta_{10} \geq 2.0,(D / H)_{P} \leq 1.6 \times 10^{-4}$ (and, for the standard model of 3 light neutrino species, $Y_{P} \geq 0.236$ ). This bound is of some interest considering the claim of a possible detection of deuterium in a QSO absorption system at a level of $(D / H)_{\mathrm{QSO}} \approx 1.9-2.5 \times 10^{-4}$ (Songaila et al. 1994; Carswell et al. 1994).

\section{Comparison With Previous Bounds}

The bounds of primordial deuterium derived above are quantitatively similar to previous bounds (YTSSO; WSSOK; ST92) but, there are some qualitative differences. One source of these differences is the new Geiss (1993) solar system abundances; another is the use of mass fractions $\left(X_{2}\right)$ rather than number ratios to hydrogen $\left(y_{2}\right)$. For example, YTSSO had derived a version of equation (8) which neglected $X_{\odot} / X_{P}(\approx 0.92)$ and related $y_{23 P}$ (rather than $y_{2 P}$; $\left.y_{2 P}<y_{23 P}\right)$ to $y_{23 \odot}$ and $y_{3 \odot}: y_{23 P}<y_{23 \odot}+\left(g_{3 \odot}^{-1}-1\right) y_{3 \odot}$. For the $2 \sigma$ upper bounds to ${ }^{3} \mathrm{He}$ and $\mathrm{D}+{ }^{3} \mathrm{He}$, YTSSO took: $y_{23 \odot} \leq 4.3 \times 10^{-5}, y_{3 \odot} \leq 1.9 \times 10^{-5}$, which leads to: $10^{5} y_{23 P}<2.4+1.9 g_{3 \odot}^{-1}$. Then, for $g_{3 \odot} \geq 1 / 4$, YTSSO derive $y_{23 P} \leq 10 \times 10^{-5}$ which, corresponds to $\eta_{10} \geq 3$ (YTSSO); actually, to two significant figures, $\eta_{10} \geq 2.8$. WSSOK used the YTSSO constraint but, slightly different $2 \sigma$ bounds to solar system $\mathrm{D}+{ }^{3} \mathrm{He}$ and ${ }^{3} \mathrm{He}$ to derive: $10^{5} y_{23 P}<3.1+1.8 g_{3 \odot}^{-1}$. For $g_{3 \odot} \geq 1 / 4$, WSSOK find $y_{23 P}<10.3 \times 10^{-5}$ (WSSOK also derive the constraint $y_{23 P} X_{P}<7.5 \times 10^{-5}$ which, for $Y_{P} \leq 0.25$, results in $y_{23 P} \leq 10 \times 10^{-5}$ ); this, too, corresponds to $\eta_{10} \geq 2.8$ (WSSOK). Finally, ST92 used the DSS stellar models for $g_{3}$ in the standard galactic evolution models of Tosi (1988) to identify the ranges of $X_{2 P}$ and $X_{3 P}$ allowed by the solar system and ISM data. For the $2 \sigma$ upper bounds to solar system abundances ST92 chose $X_{2 \odot} \leq 4.5 \times 10^{-5}$ and $X_{3 \odot} \leq 3.6 \times 10^{-5}$; for ISM deuterium they adopted $X_{2 \mathrm{ISM}} \leq 4.8 \times 10^{-5}$. In these models the effective ${ }^{3} \mathrm{He}$ survival fraction is large $\left(\Gamma_{3 \odot} \geq 1 / 2\right.$, see the earlier footnote) and the 
BBN constraint more restrictive: $X_{2 P} \leq 9.0 \times 10^{-5} ; \eta_{10} \geq 3.7$. Note that if the ST92 adopted abundances are used in eq. (10), $10^{5} X_{2 P}^{0} \leq 4.2+2.4 \Gamma_{3 \odot}^{-1}$, which, for $\Gamma_{3 \odot} \geq 1 / 4$, implies $X_{2 P}^{0} \leq 13.8 \times 10^{-5}$ and $\eta_{10}^{0} \gtrsim 2.7$; this is to be compared to $X_{2 P}^{0} \leq 16 \times 10^{-5}$ and $\eta_{10}^{0} \gtrsim 2.5$ using the Geiss (1993) abundances. Thus, although the Geiss abundances loosen the restrictions on $\eta_{10}^{0}$ compared with YTSSO and WSSOK, it is seen that the neglect of primordial ${ }^{3} \mathrm{He}$ is significant and, the Geiss abundances in eq. (9) (with $\Gamma_{3 \odot} \geq 1 / 4$ ), lead to the more restrictive constraint found above: $X_{2 P}^{\mathrm{MAX}} \leq 11 \times 10^{-5} ; \eta_{10}^{\mathrm{MIN}}>3.1$.

\section{Summary and Implications}

The primordial abundances of $\mathrm{D}$ and ${ }^{3} \mathrm{He}$ cannot be derived from solar system or ISM data (with the exception that $X_{2 P} \geq X_{2 \odot}, X_{2 P} \geq X_{2 \mathrm{ISM}}$ ) without the intervention of a model to follow the galactic evolution of these nuclides. It has been the goal here to derive a "generic" analytic expression relating primordial and galactic abundances. Equation (9) incorporates the effects (and uncertainties!) of stellar and galactic evolution in one free parameter $\Gamma_{3}$. For $\Gamma_{3 \odot} \geq 1 / 4$ (DSS) and the Geiss (1993) solar system abundances it has been found, neglecting net stellar production of ${ }^{3} \mathrm{He}$ in low mass stars (Iben 1967, Rood 1972) that, $X_{2 P}<X_{2 P}^{\mathrm{MAX}} \leq$ $11 \times 10^{-5}$. For standard BBN (WSSOK), this corresponds to $y_{2 P}<7.3 \times 10^{-5}$ and $\eta_{10}>3.1$. This provides a slightly more restrictive lower bound to the universal abundance of nucleons (e.g. compare to WSSOK where $\eta_{10} \geq 2.8$ ). For standard $\left(N_{\nu}=3\right) \mathrm{BBN}$ the lower bound to the predicted primordial mass fraction of ${ }^{4} \mathrm{He}$ increases from the WSSOK value to $Y_{\mathrm{BBN}} \geq 0.241 \pm 0.001$ as does the lower bound to primordial lithium: $(\mathrm{Li} / \mathrm{H})_{\mathrm{BBN}} \geq 1.2 \times 10^{-10}$. For $\eta_{10} \geq 3.1, \Omega_{B} \geq 0.045 h_{50}^{-2}$ so that, for $H_{0} \leq 100 \mathrm{~km} \mathrm{~s}^{-1} \mathrm{Mpc}^{-1}, \Omega_{B} \geq 0.011$. This strengthens, very slightly, the case for baryonic dark matter $\left(\Omega_{B}>\Omega_{\mathrm{LUM}}\right.$, see WSSOK).

The upper bound to $\eta$ from the lower bound to primordial $\mathrm{D}\left(X_{2 P} \geq 1.7 \times\right.$ $\left.10^{-5}, \eta_{10} \leq 9.0\right)$ is weaker than that from lithium $\left[(\mathrm{Li} / \mathrm{H})_{P} \leq 2 \times 10^{10}, \eta_{10} \leq 4.0\right]$ or from ${ }^{4} \mathrm{He}\left(Y_{P} \leq 0.243, \eta_{10} \leq 3.9\right)$. If this bound were saturated $\left(\eta_{10} \approx 9.0\right)$, the primordial lithium abundance would be $\sim$ half solar and $Y_{P} \approx 0.253 \pm 0.001$ (for $N_{\nu}=3$ ).

In closing, it must be reemphasized that the analytic framework developed here is not a replacement for detailed evolution models. In the absence of such models, the choice of the ${ }^{3} \mathrm{He}$ survival fraction, $\Gamma_{3}$, is little more than a guess. Rather, the potential value of this framework is that it provides a concise way to compare the results of different models; models which yield different constraints using the same observational data must differ in $\Gamma_{3}$. Tracing the source(s) of these differences may help to choose among different evolution models and/or to decide 
on the true uncertainties in the derived primordial abundances.

We thank Daniele Galli for calling our attention to the issue of the correct meaning of the $g_{3}$ values published by DSS and adopted by ST92. This work was begun when G. S. was an Overseas Fellow at Churchill College, Cambridge and a Visiting Fellow at the Institute of Astronomy and he thanks them for hospitality. The work at OSU is supported by DOE grant DE-FG02-94ER-40823. 


\section{References}

Boesgaard, A.M. and Steigman, G., Ann. Rev. Astron. Astrophys. 23, 319 (1985).

Carswell, R.F., Rauch, M., Weymann, R.J., Cooke, A.J. and Webb, J.K., MNRAS 268, L1 (1994).

Dearborn, D.S.P., Schramm, D.N. and Steigman, G., ApJ 302, 35 (1986). (DSS)

Edmunds, M., MNRAS 270, L37 (1994).

Galli, D., Palla, F., Straniero, O., Ferrini, F., ApJ 432, L101 (1994,)

Geiss, J., in Origin and Evolution of the Elements (eds. N. Prantzos, E. VangioniFlam and M. Cassé; Cambridge Univ. Press), p.89 (1993).

Hogan, C.J., University of Washington preprint (1994)

Iben, I., ApJ 147, 624 (1967).

Linsky, J.L., Brown, A., Gayley, K., Diplas, A., Savage, B.D., Ayres, T.R., Landsman, W., Shore, S.W. and Heap, S., ApJ 402, 694 (1993).

Olive, K.A. and Steigman, G., ApJS (In Press). (OSU-TA-6/94)

Rood, R.T., ApJ 177, 681 (1972).

Rood, R.T., Steigman, G. and Tinsley, B.M., ApJ (Lett) 207, L57 (1976).

Songaila, A., Cowie, L.L., Hogan, C. and Rugers, M., Nature 368, 599 (1994).

Steigman, G. and Tosi, M., ApJ 401, 150 (1992). (ST92)

Steigman, G., Schramm, D.N. and Gunn, J.E., Phys. Lett. B66, 202 (1977)

Steigman, G., MNRAS 269, L53 (1994).

Steigman, G. and Walker, T.P., In Preparation (1994).

Tosi, M., A\&A 197, 33 (1988).

Vangioni-Flam, E. and Audouze, J., A\&A 193, 81 (1988). 
Vangioni-Flam, E., Olive, K.A. and Prantzos, N., ApJ 427, 618 (1994).

Vangioni-Flam, E. and Cassé, M., Preprint (1994).

Walker, T.P., Steigman, G., Schramm, D.N., Olive, K.A. and Kang, H.S. ApJ 376, 51 (1991). (WSSOK)

Yang, J., Turner, M.S., Steigman, G., Schramm, D.N. and Olive, K.A. ApJ 281, 493 (1984). (YTSSO) 


\section{Figure Captions}

Figure 1a. The primordial deuterium mass fraction $\left(X_{2 P}\right)$ as a function of the present nucleon-to-photon ratio $\eta_{10}$. The solid curve $\left(X_{2 \mathrm{BBN}}\right)$ is the standard BBN prediction. The dotted curve $\left(X_{2 \mathrm{MAX}}^{2 \sigma, 0}\right)$ and the dashed curve $\left(X_{2 \mathrm{MAX}}^{2 \sigma}\right)$ are computed from equations (10) and (9) respectively using the $2 \sigma$ upper bounds to the abundances in eq. (13) for $\Gamma_{3 \odot}=1 / 4$.

Figure 1b. As in Figure 1a, for $\Gamma_{3 \odot}=1 / 2$.

Figure 2. Upper and lower bounds to primordial deuterium. The solid curve $\left(X_{2 \mathrm{BBN}}\right)$ and the dashed curve $\left(X_{2 \mathrm{MAX}}^{2 \sigma}\right)$ are as in Figure 1 . The dotted line $\left(X_{2 \mathrm{MIN}}^{2 \sigma}\right)$ is the $2 \sigma$ lower bound to $X_{2 P}$ from the ISM data (eq. 16). The restriction $X_{2 \mathrm{MIN}}^{2 \sigma} \leq X_{2 \mathrm{BBN}} \leq X_{2 \mathrm{MAX}}^{2 \sigma}$ bounds $\eta_{10}: 3.1 \leq \eta_{10} \leq 9.0$. 
This figure "fig1-1.png" is available in "png" format from: http://arxiv.org/ps/astro-ph/9502067v1 


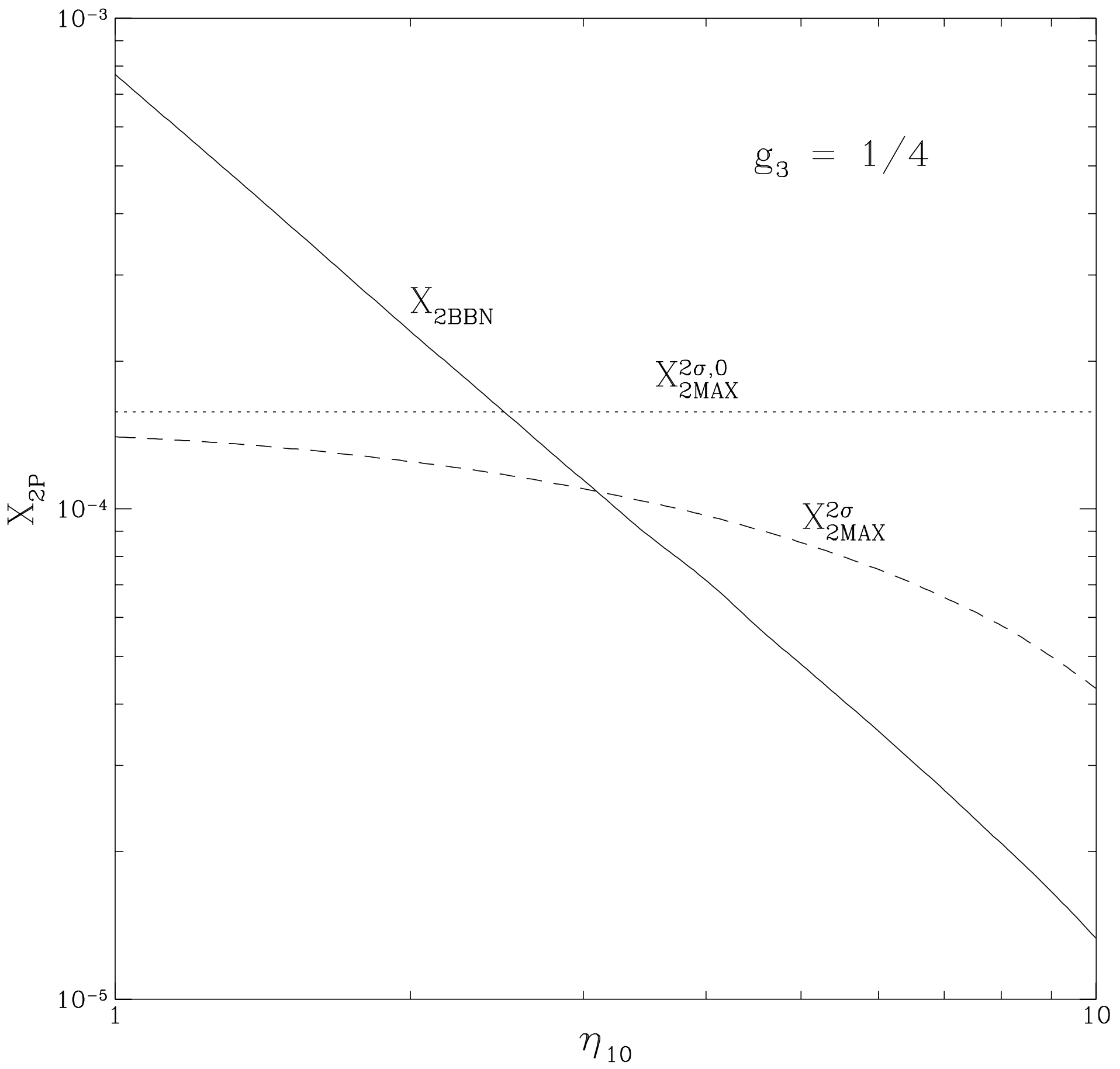




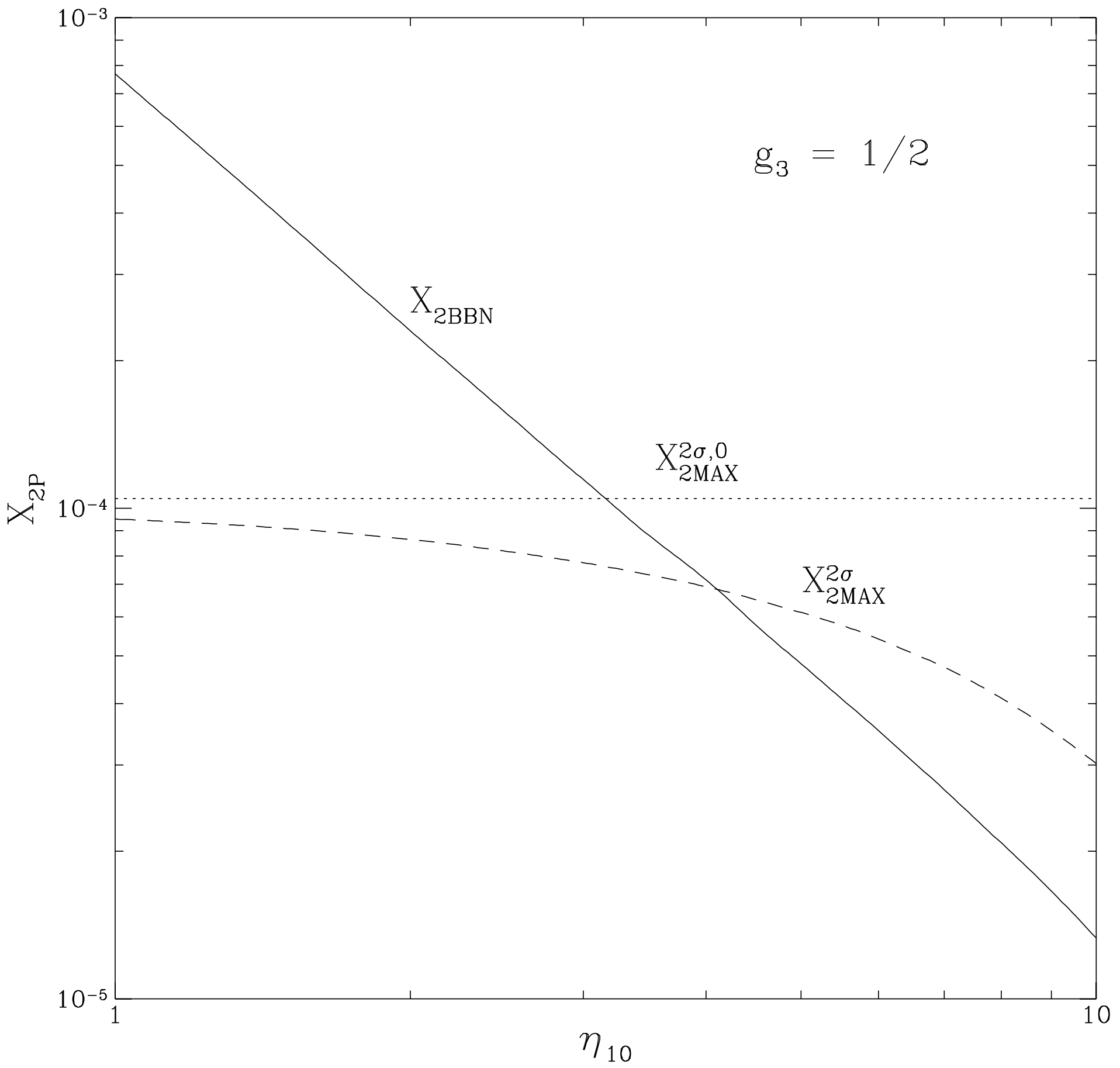


This figure "fig1-2.png" is available in "png" format from: http://arxiv.org/ps/astro-ph/9502067v1 


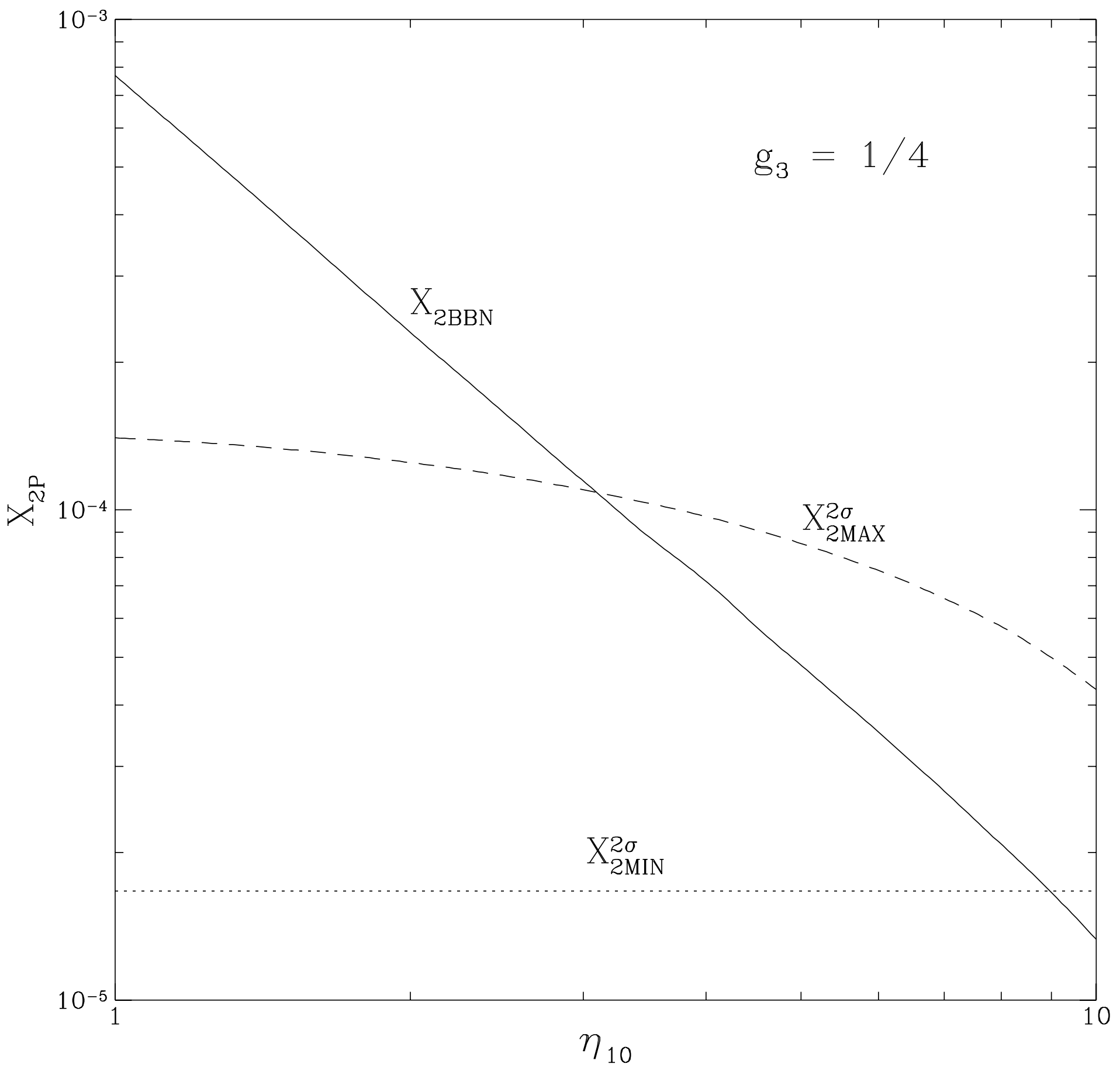


This figure "fig1-3.png" is available in "png" format from: http://arxiv.org/ps/astro-ph/9502067v1 